\title{
Corrigendum \\ Erectile dysfunction in chronic renal failure patients undergoing hemodialysis in Egypt
}

International Journal of Impotence Research (2005) 17, 390. doi:10.1038/sj.ijir.3901321

Correction to: International Journal of Impotence Research (2005) 17, 180-185. doi:10.1038/sj.ijir.3901286

Following the publication of this article, the author identified the following errors:

1. In line 13 of the abstract, 'The univariate logistic regression analysis showed that' should be omitted.

2. In the section entitled Prevalence and severity of ED:

a. The first paragraph should read:

'The prevalence of ED among the HD patients was $82.5 \%$ compared to $30 \%$ among controls. The prevalence of ED in the HD group was significantly higher than in controls $(P<0.001)$. The prevalence of ED in HD patients at the age $<50$ and $\geq 50$ y was 80 and $88 \%$, respectively, while the prevalence of ED in controls for the same age groups was 28 and $69.8 \%$ as shown in Figure 1. The prevalence of ED was significantly higher in the age groups $<50$ y $(P<0.01)$ '.

b. In the first sentence of the second paragraph, 'yet, severe ED was only significantly higher in younger age groups compared to controls' should be omitted.

3. In the section entitled Prevalence of risk factors, 'The univariate logistic regression analysis showed that' should be omitted. 